\title{
A new system for the measurement of gripping force based on scattering
}

\author{
M. Becchetti ${ }^{1}$, R. Marsili ${ }^{1}$, F. Cannella ${ }^{2}$, A. Garinei ${ }^{3}$ \\ ${ }^{1}$ Dipartimento di Ingegneria, Università degli Studi di Perugia, Perugia, 06125, Italy \\ ${ }^{2}$ Istituto Italiano di Tecnologia, Genova, 16163, Italy \\ ${ }^{3}$ DMII, Università degli Studi Guglielmo Marconi, Roma, 00193, Italy
}

\begin{abstract}
The measurement of contact pressure of human fingers is very important to understand human perceptual mechanisms, that is the main goal of most of the neuroscientific studies. It may also lead to a correct development of tactile devices and haptic systems, as they are intended to convey controllable and effective stimuli.

In this work, an optical measurement system based on Frustrated Total Internal Reflection (FTIR) is proposed for the measurement of the pressure distribution on the contact area between a human finger and a flat surface. The feasibility study performed shows that the tested sensor can be effectively used for the measurement of the fingertip contact pressure both on static and dynamic conditions.
\end{abstract}

\section{Section: RESEARCH PAPER}

Keywords: Frustrated Total Internal Reflection (FTIR); fingertip contact pressure; biomechanics

Citation: M. Becchetti, R. Marsili, F. Cannella, A. Garinei, A new system for the measure of gripping force based on the scattering, Acta IMEKO, vol. 6, no. 4, article 16, December 2017, identifier: IMEKO-ACTA-06 (2017)-04-16

Editor: Paul Regtien, Measurement Science Consultancy, The Netherlands

Received May 16, 2017; In final form December 01, 2017; Published December 2017

Copyright: @ 2017 IMEKO. This is an open-access article distributed under the terms of the Creative Commons Attribution 3.0 License, which permits unrestricted use, distribution, and reproduction in any medium, provided the original author and source are credited

Corresponding author: Roberto Marsili, e-mail: roberto.marsili@unipg.it

\section{INTRODUCTION}

The investigation of the mechanisms of human tactile perception represents a fundamental topic in haptics. The fingertip deformation is the basic mechanical action in the tactile perception, since tactile sensitivity depends on the tissue strain and hence on the contact area. Different models are available to predict the behaviour of the fingertip in terms of contact area, deformation and pressure distribution [1], [2]. Measurements are thus required both to determine the static fingertip area and force as input to the numerical models and to measure the area and the pressure necessary for numerical model validation. When measuring the contact pressure, the goal is to set up a measurement system with high sensitivity at low pressure, since, for specific applications, the typical pressure range of interest is from 0 to $50 \mathrm{kPa}$.

The main objective of this work is the development of a measurement system, which allows to characterize the different aspects of the interaction finger - contact surface, through the measurement of the contact area and of the pressure distribution, both on static and dynamic conditions.

Many sensors based on different physical principles have been proposed for the measurement of pressure at contact surface between two rigid or flexible bodies. Some contact pressure measurement systems have been used for measuring pressure distribution at interfaces between objects, with application to comfort analysis and improvement of vehicle seats [3], or steering wheel [4], for various biomedical applications such as measurement of contact pressure between hand and handle of a tool in order to analyse vibration transmission to the hand [5], [6] or between foot and ground for plantar pressure analysis [7], [8]. In [9]-[11] a new measurement technique for the measurement of contact pressure distributions between bodies in contact is proposed, based on the measurement principle known as thermoelasticity. Many applications have been developed [12]-[14] also in other fields such as robot technology (tactile sensors), for contact force mapping of mechanical parts. The film sensors used for those applications are based on piezo-polymers, capacitive 
sensors, conductive ink, resistive polymers sensing elements or magnetoresistivity [15]. However, many metrological problems remain unresolved, due to non-linearity, material's rheological behaviour, mounting surface curvature and shape, dynamic characteristics, etc. Also frequency limitations can be due to the sensor itself or to the electronic processing and data acquisition system, when a matrix of many sensing elements is necessary. Piezo-polymers have probably the best metrological characteristic but cannot measure very slow fluctuations or static pressure components. Sensors based on conductive ink or resistive polymers showed from preliminary tests that metrological characteristics change with time and have large hysteresis. The capacitive sensing elements can be used to measure both dynamic and static pressure with acceptable linearity and hysteresis, and bendable sensors may be applied to the wearable health monitoring/sensing system or electronic skins, as in [16]. Here an optical measurement system based on light scattering is developed and analysed. The outline of this paper is as follows. First, the design of an optimized measurement system based on Frustrated Total Internal Reflection (FTIR) is described. Then the results of specific tests performed on the measurement system are shown, to define its static and dynamic behaviour and to evaluate if the proposed measurement technique can be effectively used to measure human fingertip mechanical properties in terms of contact area and pressure distribution.

\section{EXPERIMENTAL BACKGROUND AND SETUP}

The principle of the measurement system is the light reflection/refraction at the interface between two transparent media. When the light passes through an interface between media of different refractive indices, the path of travel changes (Snell's Law).

Thus when a light source is installed at the edge of a thin glass plate and the incident angle $\vartheta_{1}$ exceeds the critical angle $\vartheta_{\text {critical }}$ (Figure 1a), the plate acts as a light guide. In this case (total internal reflection) the light cannot be seen when observing the plate from below the plate.

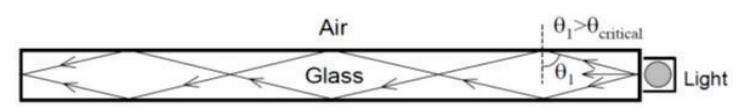

(a)

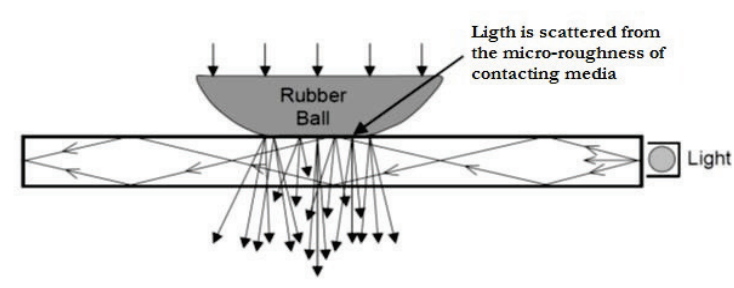

(b)

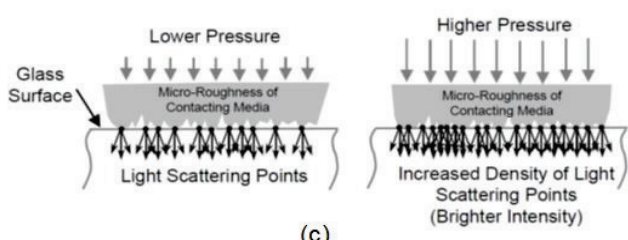

(c)

Figure 1. Principle of measurement [17]. (a) shows the lighting system, (b) the light scattering, (c) the detail of the contact area.
If a reflecting media is pressed against the plate, the surface of the media will scatter light in the contact surface, being the media with a refractive index different from the refractive index of the air (Figure 1b). By observing the scattered light, it is thus possible to view the shape of the contact surface. Depending on the roughness of the contact surface, the contact area will increase with the contact pressure, thus the light intensity can be used to measure the contact pressure between the two media (Figure 1c).

Methods based on white light refraction are used for the measurement of normal contact stress between 'soft' structures and a rigid planar surface, specifically for application to tire footprint analysis [17], [18]. These systems are optimized to measure tire footprint pressures and are not suitable for the measurement of low pressures. The design of the proposed measurement system was developed to permit the measurement of low pressures that characterize human fingertip contact. The roughness and the mechanical characteristics of the contact surface are the most critical parameters for the realization of an effective pressure measurement system based on FTIR. These parameters must be carefully evaluated, because of their effects on the range and on the sensitivity of the measurement system.

The measurement system setup is shown in Figure 2: a sliding system realized through threaded rods permits to adjust the camera position depending on its optics; a $200 \mathrm{~mm}$ x 200 $\mathrm{mm} \times 200 \mathrm{~mm}$ darkroom was realized to avoid interference of light from the external environment; a $150 \mathrm{~mm} \times 150 \mathrm{~mm} \times 10$ $\mathrm{mm}$ glass plate was fixed on a square support with a green LED strip installed around the perimeter.

The main characteristics of the glass plate are shown in Table 1.

\section{SYSTEM CHARACTERIZATION}

\subsection{Static characterization}

A first static analysis of the system was performed to characterize the behaviour of the sensor when a constant pressure is applied on it. For this purpose, the glass plate was covered with a thin membrane and fixed on a square support

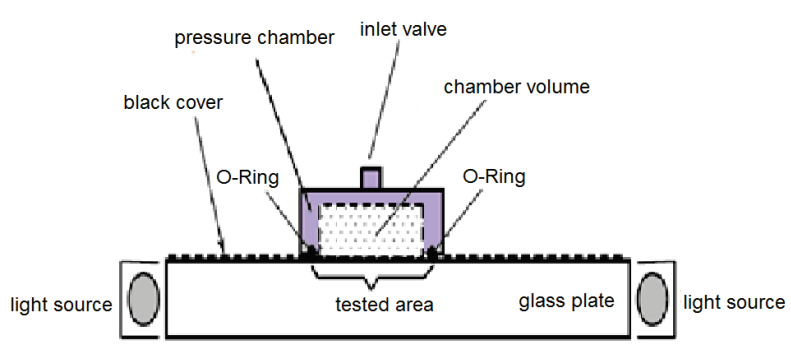

Figure 2. Test bench for static calibration.

Table 1. Glass plate characteristics.

\begin{tabular}{|l|l|}
\hline Chemical glass composition & $\left(\mathrm{SiO}_{2}\right)+$ oxides \\
\hline Aspect & Transparent \\
\hline Crystallinity & Amorphous \\
\hline Refractive index & From 1.458 to 1.86 \\
\hline Nominal tensile strength & $4 \mathrm{MPa}$ \\
\hline Nominal compression strength & $1 \mathrm{GPa}$ \\
\hline Nominal bending strength & $40 \mathrm{MPa}$ \\
\hline
\end{tabular}


with a green LED strip installed around the perimeter.

A Plexiglas frame was then realized to install the thin lattice membrane at the top of the glass plate: the membrane is stretched, thus there is a thin air gap (no contact) between the membrane and the glass plate in the starting configuration. The lattice membrane is talc-covered to increase the roughness of the contact surface and thus the sensitivity of the measurement system. Different test setups can be achieved by varying the amount (thickness) of talc powder (estimated by weighing) and using talc with different granulometry. In the work, we want to develop a methodology for measuring the metrological performance of the sensor, which is obtained by calibration. Problems like the uniformity of the overlay will be faced during prototype engineering, with a productive industrial process more accurate than a laboratory production. In addition to talcum, other materials can be used.

Figure 3 illustrates a typical result of the relation between thickness and pressure for the latex membrane used.

From this result it is possible to see, in general, the nonlinear behavior of the latex membrane. However, in the pressure range of interest $(0-50 \mathrm{kPa})$, as a first approximation, the curve pressure vs thickness reduction can be considered linear.

For the static calibration, a reference pressure is applied to the sensor by the thin membrane, which is loaded by a constant pressure chamber, whose pressure is measured by a pressure sensor connected to a Scandura Pascal 100 system. In order to avoid pressure losses, the contact between the pressure chamber and the glass plate is realized through an O-Ring for pneumatic applications (Figure 2).

The reference pressure varies from $0 \mathrm{~Pa}$ to $100 \mathrm{kPa}$, that is the maximum pressure to avoid fracture of the glass plate.

A high performance reflex digital camera (Canon EOS 1100D, with a 12.2 megapixel CMOS sensor, fast shutter speed $1 / 4000 \mathrm{~s})$, was used to take high resolution grey-level images. The camera was equipped with a $18-55 \mathrm{~mm} \mathrm{f/3.5-5.6} \mathrm{lens:} \mathrm{the}$ distance from the camera to the glass plate was adjusted depending on the focal length of the camera. The camera parameters (ISO settings, shutter speed, white balance) were adjusted to obtain maximum sensitivity for the specific setup (fnumber $=5.6$, exposure time $=1 / 8 \mathrm{~s}$, ISO 400).

The raw images (4272 x 2848 pixels) were acquired with the software Canon Eos Utility, converted in .tiff format and thus post-processed with a Matlab toolbox. Images were acquired for each pressure step (5000 $\mathrm{Pa}-21$ steps) in the calibration range.

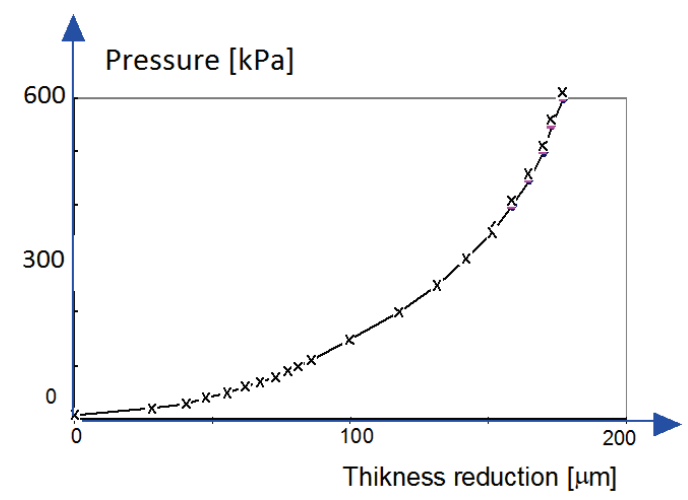

Figure 3. Typical pressure - sensor thickness reduction curve [19].
For a given pressure, the grey level of each pixel varies depending on the pixel location and on the local characteristics of the contact surface (roughness). Figure 4 illustrates a typical static calibration diagram: abscissa refers to the reference pressure, estimated by a Wika Pascal 100, with range $0-2.5$ bar, resolution 0.01 mbar, uncertainty $0.025 \%$ F.S.; the ordinate refers to the grey level (range $0 \div 256$ ) measured by the sensor. The calibration curve is obtained using the method of least squares. The uncertainty is less than $1 \%$ full scale.

After these first measurements, a measurement area was defined to reduce the dispersion due to the localized imperfections of the contact surfaces (Figure 5). This is the most effective sensing area both for static and dynamic measurements. The optimal measurement area is a $20 \times 20 \mathrm{~mm}$ box.

\subsection{Preliminary dynamic characterization}

Then a dynamic characterization was performed by loading the sensor with a sinusoidal excitation applied through a rigid feeler pin (Figure 6). The compression force, generated by the electrodynamic exciter, is measured by a load cell. The deformation of the glass plate was so small that its effect can be neglected.

Tests were performed using sine excitation in the linear range of the sensor $(10-100 \mathrm{kPa})$ : typical signals obtained are shown in Figure 7.

For the image processing specific software in LabView has been developed (Figure 8).

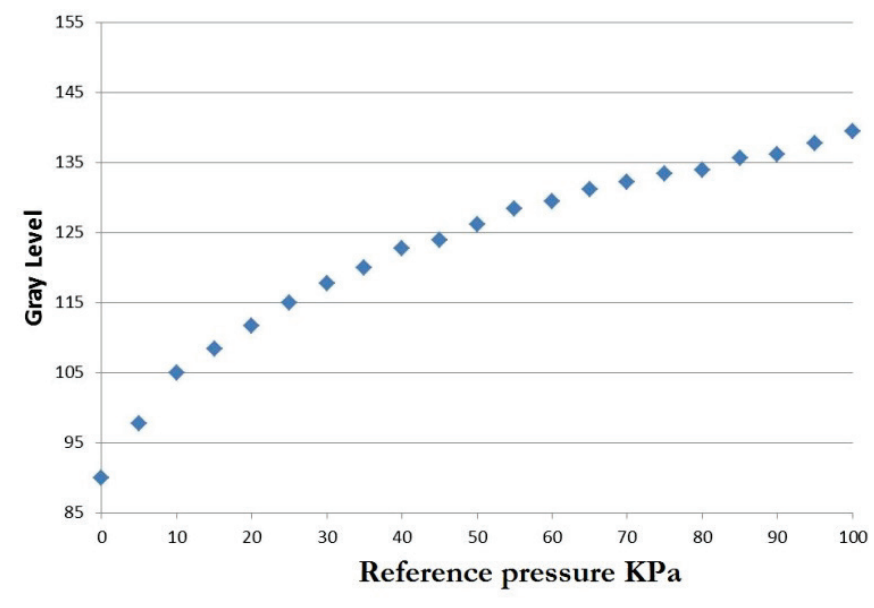

Figure 4. Typical static calibration diagram for a pixel.

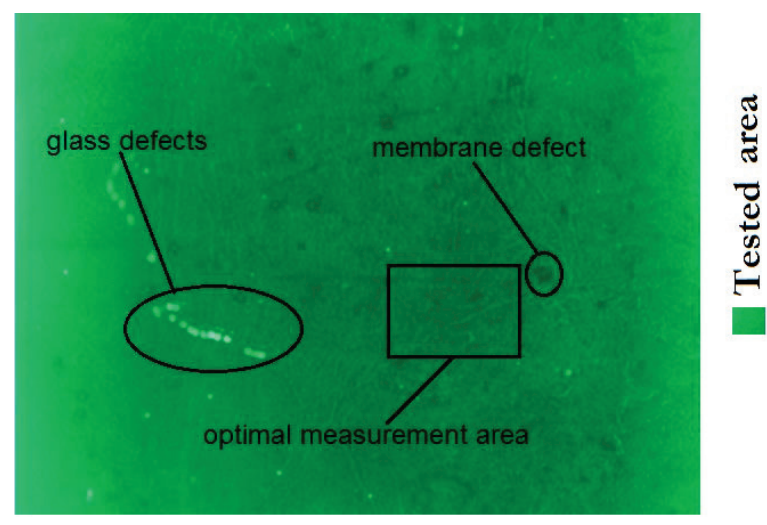

Figure 5. Measurement area selection. 


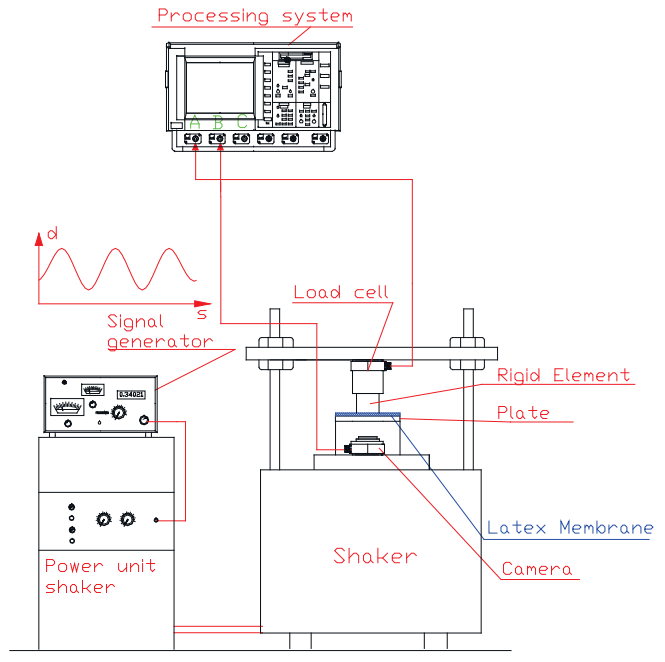

Figure 6. Test bench for dynamic characterization.

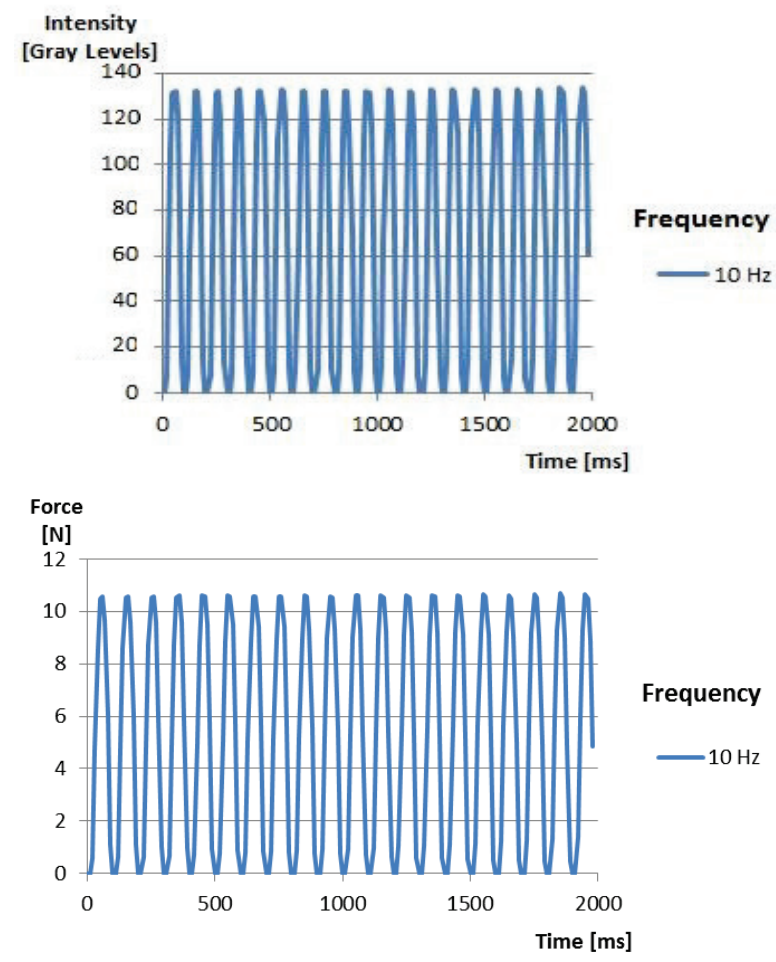

Figure 7. Time history output system and force.Figure x. Stamp.

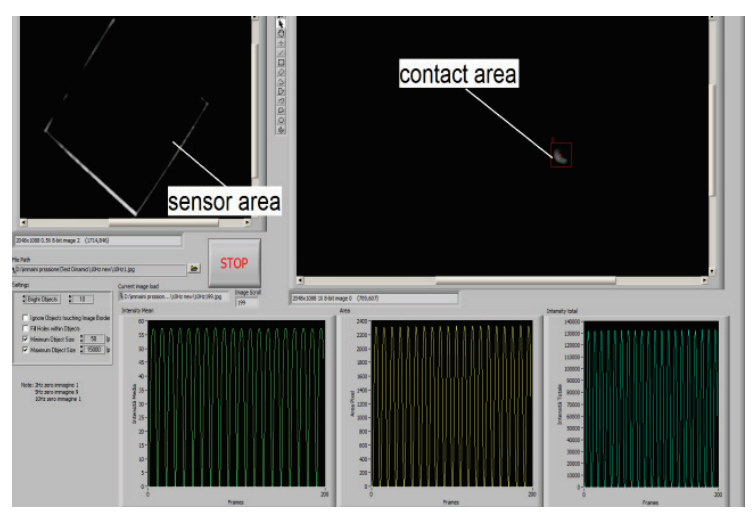

Figure 8. Image processing software interface.
The tests are conducted changing the excitation frequency, while keeping the level of force fixed. The ratio between system output (gray level) and force amplitude vs frequency is plotted in Figure 9.

These results allow establishing that a useful bandwidth is from 0 up to $10 \mathrm{~Hz}$ with tolerable response decrease lower than $5 \%$.

Further tests are planned to determine the maximum measurable frequency using this system.

\section{CONCLUSIONS}

The analysis of the contact pressure between the finger and a contact surface is of remarkable interest in the field of biomedical design, to characterize human fingertip mechanical properties in terms of contact area, deformation and pressure distribution.

In this work a measurement technique based on FTIR is proposed for the analysis of contact pressure between the finger and a flat contact surface (glass plate).

A FTIR measurement system was designed and tested to evaluate the feasibility and effectiveness of this measurement technique to measure the contact area and the pressure necessary for numerical fingertip model validation.

Results showed that there are good assumptions to use the sensor for the measurement of the fingertip contact pressure both on static and dynamic conditions.

Consistency and repeatability observed at this first stage is very encouraging for the development of an effective fingertip pressure measurement system based on FTIR.

This will permit to analyse the behaviour of the finger for different conditions, and thus to validate numerical models not only in contact area and force, but also in pressure, that is suitable for investigating the fingertip deformation on static and dynamic conditions.

Plans for future work are to further explore new materials for a higher sensitivity and testing different configurations with a complete set of loading conditions.

\section{REFERENCES}

[1] J. Z. Wu, R. G. Dong, S. Rakheja, A. W. Schopper, and W. P. Smutz, "A structural fingertip model for simulating of the biomechanics of tactile sensation," Med. Eng. Phys., Mar. 2004, vol. 26, no. 2, pp. 165-175.

[2] S. Shimawaki, N. Sakai, "Quasi-static deformation analysis of a human finger using a three-dimensional Finite Element Model

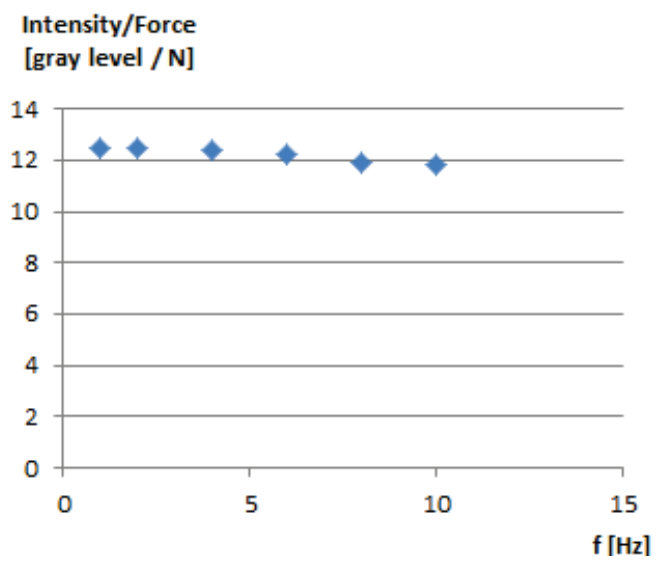

Figure 9. Time history output system and force. 
Constructed from CT Images", Journal of Environment and Engineering, 2007, Vol. 2, no. 1.

[3] M. Shiratori, M. Ishida, "Development of body pressure distribution measurement device", JSAE Review, July 1992, Vol. 13 n. 3.

[4] R. Marsili, A. Garinei, "Development of a new capacitive matrix for pressure distribution measurement" International Journal of Industrial Ergonomics Volume 44, Issue 1, January 2014, Pages 114-119 doi: 10.1016/j.ergon.2013.11.012.

[5] R. Marsili, G.L. Rossi, "The measurement of contact and grip force as reference for human hand transmitted vibration evaluation by laser scanning vibrometers", II Int. Conf. on Vibration Measurements by Laser Techniques, 23-25 Sept. 1996, Ancona Italy, SPIE Vol. 2868.

[6] R. Gurram, G.J. Gouw, S. Rakheja, "Grip pressure distribution under static and dynamic loading", Int. Jou. of Experimental Mechanics.

[7] P. Sanfilippo, R. Stress, K. Moss. "Dynamic plantar pressure analysis", Jou. of the American Pediatric Medical Associations, 1992; Vol. 82 n. 10 pp 507-514.

[8] 8 A. Mair, W. Diebschlag, O. Distl, H. Krausslich, "Measuring device for the analysis of pressure distribution on the foot soles of cattle", J. Vet. Med, 1988; A 35 pp 696-707.

[9] M. Becchetti, R. Flori, R.. Marsili, M. Moretti, "Comparison between digital image correlation and thermoelasticity for strain field analysis" (2010) 9th International Conference on Vibration Measurements by Laser and Noncontact Techniques and Short Course; Ancona; Italy; 22 - 25 June 2010 AIP Conference Proceedings, Volume 1253, pp. 233-240, doi=10.1063/1.3455462 ISSN: 0094243X ISBN: 978073540802-9.

[10] R. Marsili, G. Brustenga, M. Moretti, J. Pirisinu, G.L. Rossi, "Measurement on mechanical component by thermoelasticity", Journal Applied Mechanics and Materials, ISBN 0-87849-987-3 ISSN 1660-9336, Vols 3-4, August 2005, pp 337-342, pp 411 416. SCOPUS: 2-s2.0-33745170726.

[11] Jean-Francois Brouckaert, Roberto Marsili, Gianluca Rossi, "Development and experimental characterization of a new contact sensor for blade tip timing", 10th International Conference on Vibration Measurements by Laser and Noncontact Techniques - AIVELA, Ancona, ITALY, JUN $27-$
29, 2012 Conference Proceedings Volume 1457 Pages: 61 - 68 DOI: 10.1063/1.4730543 Published: 2012 IDS Number: BBI34 ISSN: 0094-243X ISBN: 978-0-7354-1059-6 SCOPUS: 2s2.0-84874468369.

[12] R. Marsili, A. Garinei, "Design of an optical measurement system for dynamic testing", Measurement Volume 46, Issue 5, June (2013), pp. 1715-1721 DOI information: 10.1016/j.measurement.2013.01.006, ISSN: 0263-2241, Elsevier Science.

[13] R. Marsili, A. Garinei, "A new diagnostic technique for ball screw actuators", Measurement, Volume 45, issue 5 (June, 2012), p. 819-828, ISSN: 0263-2241 DOI: 10.1016/j. measurement. 2012.02.23, Elsevier Science.

[14] R. Marsili, M. Moretti, G.L. Rossi, "Thermoelastic Modal Stress Analysis", IMAC XXVI Conference \& Exposition on Structural Dynamic, Orlando, Florida USA, 4 - 7 February 2008; ISBN: 0912053984 ISBN 9781605600666; SCOPUS : 2-s2.084861536363.

[15] E. Cardelli, A. Faba, R. Marsili, G. Rossi, R. Tomassini, "Magnetic nondestructive testing of blade tips", Journal of Applied Physics 117, 17A705 (2015); doi: 10.1063/1.4907180.

[16] R. Marsili, A. Garinei, "Measurement of pressure distribution on a membrane of a pump for biomedical applications through capacitive film sensors" Measurement: Journal of the International Measurement Confederation, 55, pp. 110-116 (2014) DOI: 10.1016/j.measurement.2014.04.040.

[17] S.Begej, "Planar and finger-shaped optical tactile sensors for robotic applications", IEEE J. Robotics and Automation, 4, 5 (Oct. 1988), p.472-484.

[18] A. N. Gent, J. D. Walter, "The Pneumatic Tire", The National Highway Traffic Safety Administration, U.S. Department of Transportation, Washington DC 20590, August 2005, pp 248250.

[19] R. Marsili, A. Garinei, "Thermoelastic Stress Analysis of the Contact Between a Flat Plate and a Cylinder", Measurement: Journal of the International Measurement Confederation, 52 (1), pp. 102-110 (2014)

http://dx.doi.org/10.1016/j.measurement.2014.03.005 (2014). 